\title{
Molecular Dynamics Simulation of Crack Propagation in Single-Crystal Aluminum Plate with Central Cracks
}

\author{
Jun Ding, Lu-sheng Wang, Kun Song, Bo Liu, and Xia Huang \\ College of Mechanical Engineering, Chongqing University of Technology, Chongqing 400054, China \\ Correspondence should be addressed to Xia Huang; huangxia@cqut.edu.cn
}

Received 21 April 2017; Accepted 20 June 2017; Published 27 July 2017

Academic Editor: Jean M. Greneche

Copyright (C) 2017 Jun Ding et al. This is an open access article distributed under the Creative Commons Attribution License, which permits unrestricted use, distribution, and reproduction in any medium, provided the original work is properly cited.

\begin{abstract}
The crack propagation process in single-crystal aluminum plate (SCAP) with central cracks under tensile load was simulated by molecular dynamics method. Further, the effects of model size, crack length, temperature, and strain rate on strength of SCAP and crack growth were comprehensively investigated. The results showed that, with the increase of the model size, crack length, and strain rate, the plastic yield point of SCAP occurred in advance, the limit stress of plastic yield decreased, and the plastic deformability of material increased, but the temperature had less effect and sensitivity on the strength and crack propagation of SCAP. The model size affected the plastic deformation and crack growth of the material. Specifically, at small scale, the plastic deformation and crack propagation in SCAP are mainly affected through dislocation multiplication and slip. However, the plastic deformation and crack propagation are obviously affected by dislocation multiplication and twinning in larger scale.
\end{abstract}

\section{Introduction}

With the development of micro/nanoelectromechanical systems, the mechanical properties of metal materials at the nanoscale have attracted significant attention. Nanoplate is the basic structure of micro/nanodevices. However, being attributed to the different heat-treatment processes, defects such as microcracks and microvoids are formed inevitably, which affect the mechanical properties of the nanoplate. Besides, the mechanical properties of nanoplates can also be influenced by factors such as size, temperature, and strain rate [1].

Defects such as microcracks and microvoids have obvious effects on the mechanical properties and crack propagation in the nanoplates. White [2] simulated the crack propagation process in nanoaluminum with microcracks under uniaxial tension and cyclic load by molecular dynamics method. They found that the propagation of microcracks was related to emission and slippage of dislocations, and plastic hardening was induced at crack tips due to dislocation pileup and intersecting. Yuan et al. [3] established the molecular dynamics model of single-crystal aluminum plate (SCAP) with voids under high tension strain rate, discussed the effect of void radius on stress-strain relationship, and observed the void evolution and aggregation process. However, the effects of dislocation, twin crystal, and phase transition on void extension and fusion have not been analyzed till date. Through molecular dynamics simulation of the single-crystal model with single-edge crack under uniaxial tension, Cui and Beom [4] observed the propagation process of single-edge crack and the concurrent phenomena including twin crystal and dislocation and further analyzed the effect of crack length on stress-strain relationship. However, the mode of influence of the microscopic mechanisms including dislocation and twin crystal on the crack propagation was not explained. Chen [5] observed the fracture failure process in industrial steelgrit bainite structure with microcracks under uniaxial tension by scanning electron microscopy (SEM), and the results revealed that the major fracture mechanism involved the formation, growth, and connection of microvoids. Takahashi et al. [6] observed the crack propagation process in aluminum alloy under fatigue load and reached the conclusion that the crack propagation was intensively anisotropic, and the crack propagation and atom slippage resulted in decrease in the stress of the material. Shanyavskiy [7] investigated the failure processes of aluminum alloys under cyclic load at 
various scales and found that the plastic instability in loading direction at micro/nanoscales resulted in the generation of surfaces cracks, thus affecting the mechanical properties of aluminum alloys.

On the other side, the model size has an important effect on the strength and crack propagation in materials. Mahato et al. [8] investigated the effects of model size and strain rate on the stress-strain relationship of single-crystal copper at the nanoscale by molecular dynamics method and obtained the critical strain rate of single-crystal copper. They believed that dislocation nucleation was the major mechanism responsible for the material fracture. Potirniche et al. [9] studied the void growth and coalescence in single-crystal nickel and discussed the effects of atom number, strain rate, and temperature on plastic deformation and void extension; however, the effect of dislocations on void growth and condensation had not been discussed. Dunstan and Bushby [10] investigated the effect of model size on strength of metal with facecentered cubic (FCC) structure and found that the strength was inversely proportional to the size, and different sizes have different spaces for starting dislocation sources. Yaghoobi and Voyiadjis [11] studied the size effect of cylindrical nanocopper under compressive load by nanoindentation method and found that the dislocation was the major mechanism affecting the strength of the material. It is a pity that the effect of dislocation on crack propagation had not been discussed in papers.

Temperature and strain rate are also important factors affecting the mechanical properties and crack propagation of materials. Liao et al. [12] investigated the temperature effect on stress-strain curve of SCAP with voids obtained in the temperature range $300-1200 \mathrm{~K}$ under aging conditions. Nevertheless, the influence of micromechanism such as dislocation on stress-strain curve had not been analyzed. Shiari and Miller [13] studied the fracture failure process of metal materials with FCC structure with low temperatures, found that brittle fracture occurred for nanomaterials during low temperatures, and believed that temperature is the key factor affecting critical crack propagation velocity. Horstemeyer et al. [14] simulated the fracture failure process of singlecrystal FCC metals under tensile load by molecular dynamics method for various strain rates (in the range $10^{7}-10^{12} \mathrm{~s}^{-1}$ ) and found that the material was of obvious sensibility for strain rate. Yan et al. [15] studied the strain rate sensibility of aluminum and aluminum alloys by experiments. They divided the sensitive range of strain rate into three regions; unfortunately, the effects of microscopic mechanisms such as dislocation on the stress-strain relationship were not discussed.

Although numerous high-level researches have been carried out on factors affecting the mechanical properties of metal materials with microcracks under tensile load, deficiently, the effect of microscopic mechanism of dislocation and twinning on stress-strain relationship and crack propagation has rarely been reported. In this study, the crack propagation process in nanoaluminum plate with central cracks under tensile load was studied by molecular dynamics method; the effects of model size, crack length, temperature, and loading strain rate on material strength and crack propagation were systematically analyzed. Further the impacts of

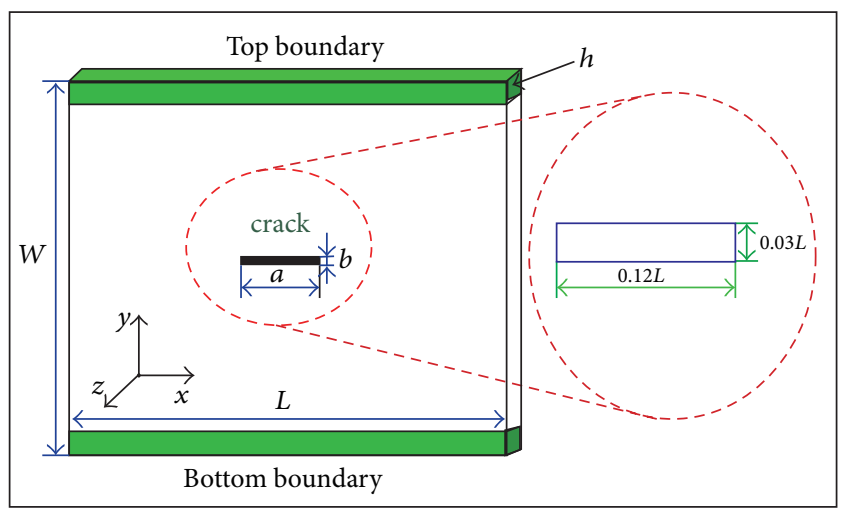

FIGURE 1: Geometry models of single-crystal aluminum plate with a central crack in y direction under tension. The $x$-, $y$-, and $z$-axis are along [100], [010], and [001] orientations, respectively, representing the main directions of lattice in single-crystal aluminum cell.

dislocation, twin crystal, and phase transition on material's mechanical properties and crack propagation were discussed.

\section{Molecular Dynamics Simulation}

2.1. Establishment of Model. Figure 1 shows the schematic representation of the model, in which the positive directions corresponding to $x-, y$-, and $z$-axis are [100], [010], and [001] orientations, respectively, representing the main directions of lattice in single-crystal aluminum cell. $L$ and $W$ represent the length and height of the model, respectively, and the ratio $L / W$ is $1 . h$ is the thickness of aluminum plate, and its value is $1.62 \mathrm{~nm}$, which is more than twice the cutoff radius of the selected potential function to avoid boundary effects of $z$ axis. There is a crack in the center of the plate with the length $a$ of $0.12 L$ and the width $b$ of $0.03 L$. In order to establish the model under uniaxial tension, an atomic layer with rigid atoms was set at the boundary of $y$-axis, and the thickness of boundary-layer atoms was equal to the lattice constant. Velocity load was applied to the boundary-layer atoms along the positive and negative directions of $y$-axis, respectively. The $x$ - and $y$-boundary are free boundary conditions, and $z$ boundary is periodic boundary condition.

Table 1 shows the parameters of the different atomic models, including plate length and width $L$ and $W / \mathrm{nm}$, crack lengths and width $a$ and $b / \mathrm{nm}$, atomic number $N$, temperatures $T / \mathrm{K}$, and loading strain rates $R / \mathrm{s}^{-1}$. To investigate the atomic numbers effect of SCAP under tensile load, the volume fraction of crack size in the model was kept constant as Potirniche's model of single nickel [14]. The deformation temperature was $300 \mathrm{~K}$ and the strain rate was $10^{9} \mathrm{~s}^{-1}$. Four models (model 1 4) were established with atom numbers from 6704 (the minimum) to 410760 (the maximum), and the length sizes of all models were in the range of $1-100 \mathrm{~nm}$. Liu et al. [16] set the maximum crack length as $0.25 \mathrm{~L}$ in the study on effect of crack length on strength and crack propagation in single-crystal aluminum with single-edge cracks. To investigate the effects of crack length on strength and crack propagation in SCAP, under the conditions with 
TABLE 1: The parameters of the different atomic models, including plate length and width $L$ and $W / \mathrm{nm}$, crack length and width $a$ and $b / \mathrm{nm}$, atomic number $N$, deformation temperatures $T / K$, and loading strain rates $R / \mathrm{s}^{-1}$.

\begin{tabular}{|c|c|c|c|c|c|c|c|}
\hline Model & $L$ & $W$ & $a$ & $b$ & $N$ & $T$ & $R$ \\
\hline Case 1 & 8.1 & 8.1 & 0.972 & 0.243 & 6704 & 300 & $10^{9}$ \\
\hline Case 2 & 16.2 & 16.2 & 1.944 & 0.486 & 26136 & 300 & $10^{9}$ \\
\hline Case 3 & 32.4 & 32.4 & 3.888 & 0.972 & 103304 & 300 & $10^{9}$ \\
\hline Case 4 & 64.8 & 64.8 & 7.776 & 1.944 & 411784 & 300 & $10^{9}$ \\
\hline Case 5 & 32.4 & 32.4 & 2.592 & 0.648 & 103304 & 300 & $10^{9}$ \\
\hline Case 6 & 32.4 & 32.4 & 6.480 & 1.620 & 103304 & 300 & $10^{9}$ \\
\hline Case 7 & 32.4 & 32.4 & 3.888 & 0.972 & 103304 & 600 & $10^{9}$ \\
\hline Case 8 & 32.4 & 32.4 & 3.888 & 0.972 & 103304 & 900 & $10^{9}$ \\
\hline Case 9 & 32.4 & 32.4 & 3.888 & 0.972 & 103304 & 300 & $10^{8}$ \\
\hline Case 10 & 32.4 & 32.4 & 3.888 & 0.972 & 103304 & 300 & $10^{10}$ \\
\hline
\end{tabular}

the model size of 103304, the deformation temperature of $300 \mathrm{~K}$, and the loading strain rate of $10^{9} \mathrm{~s}^{-1}$, two models with crack length of $2.592 \mathrm{~nm}$ (model 5) and $6.48 \mathrm{~nm}$ (model 6) were established. Noteworthily, different crack lengths result in the change of atom number in model systems. However, the change rate with respect to the total atom number was calculated to be only $0.38 \%$; thus the effect of the change of crack length on the total atom number was negligible [14]. To investigate the effect of deformation temperature on strength and crack propagation in SCAP, two models with temperature of $600 \mathrm{~K}$ (the recrystallization temperature) and $900 \mathrm{~K}$ (high temperature, the melting point of aluminum is about $950 \mathrm{~K}$ ) were established (labeled as models 7 and 8 , resp.). Moreover, the atom number was 103304, the crack length was $3.888 \mathrm{~nm}$, and the loading strain rate was $10^{9} \mathrm{~s}^{-1}$. To investigate the effect of loading strain rate on strength of SCAP, the loading strain rates were set as $10^{8} \mathrm{~s}^{-1}$ (model 9) and $10^{10} \mathrm{~s}^{-1}$ (model 10), respectively. The temperature was $300 \mathrm{~K}$ and the crack length was $3.888 \mathrm{~nm}$.

\subsection{Numerical Integration Algorithm and Characterization}

Methods. The Velocity-Verlet algorithm was applied for the numerical integration solution of kinematic equation [20]. Figure 2 shows the deductive schematic representation for iterative deduction of Velocity-Verlet algorithm, which has the advantages of high computation speed and high computational accuracy. When the location, velocity, and acceleration at time $t$ are known, the location at time $t+\Delta t$ can be obtained, through which the acceleration at time $t+\Delta t$ can also be obtained. Finally, the velocity at time $t+\Delta t$ can be attained by using the velocity and acceleration at time $t$ and acceleration at time $t+\Delta t$.

To characterize the microscopic phenomena such as dislocation, twin crystal, and phase transition in material during the tensile process, the center-symmetry parameter method (CSP) [21] was utilized to recognize the dislocations among atoms, and the common neighbor analysis (CNA) was performed to characterize the change of crystal structure $[22,23]$. The center-symmetry parameter of atom $j$ is as follows:

$$
\rho_{j}=\sum_{j=1}^{6}\left|\vec{R}_{j}+\vec{R}_{j+6}\right|^{2}
$$

TABLE 2: The definition for defects recognition in centrosymmetry parameter (CSP) of Face-Center Cubic (FCC) structure.

\begin{tabular}{lcc}
\hline Structure & CSP & Colors \\
\hline Perfect & $C<3$ & Blue \\
Dislocation & $3<C<9$ & Green \\
Surface atoms & $9<C<12$ & Red \\
\hline
\end{tabular}

where $\vec{R}_{j}$ and $\vec{R}_{j+6}$ are six pairs of vectors of the relatively nearest atoms in FCC single-crystal aluminum. $\rho_{j}$ is the center-symmetry parameter of atom $j$. The definition of crystal structure defects is shown in Table 2.

2.3. Selection and Verification of Potential Function. In this study, the embedded atom method (EAM) potentials [24, 25] were employed to describe and characterize the interaction between atoms in single-crystal aluminum, and the basic form of the EAM potential function is as follows:

$$
\begin{aligned}
& U=\sum_{i} F_{i}\left(\rho_{i}\right)+\frac{1}{2} \sum_{j \neq i} \phi_{i j}\left(r_{i j}\right), \\
& \rho_{i}=\sum_{j \neq i} \rho_{j}\left(r_{i j}\right),
\end{aligned}
$$

where $U$ is the total energy of the system, which is composed of two parts including an embedded energy, that is, $F_{i}\left(\rho_{i}\right)$ in formula (2), and a pair potential that represents the interaction between atoms. $\rho_{i}$ is the sum of electron cloud density at the $i$ th atom generated by the extranuclear electrons of other atoms. $r_{i j}$ is the distance between the $i$ th and the $j$ th atoms, and $\rho_{j}\left(r_{i j}\right)$ is the fitted distribution function of electron density.

To verify the accuracy of the embedded atom potential function, the fundamental physical quantities such as lattice constant, elastic constant, and melting point of the potential function were calculated in this study, and the calculated results were verified through comparison with the experimental results [17-19], as listed in Table 3. The calculated results of the lattice constant, elastic constant, and melting point of this potential function were found to be in good agreement with the experimental results, indicating that this potential function is applicable to be analyzed by this model. 

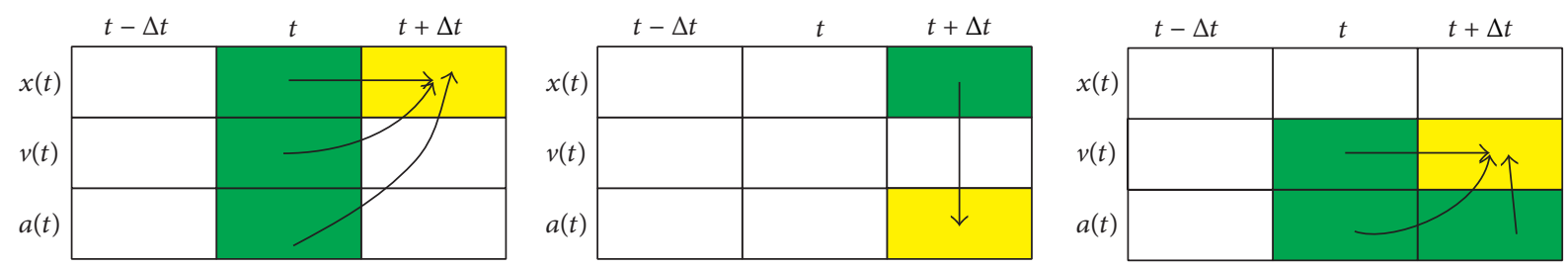

FIGURE 2: Deduction process of the Velocity-Verlet algorithm.

TABLE 3: Comparison of calculation and experiments for aluminum potential, including lattice parameters $a / \AA$, elastic constants $C_{11}$, $C_{12}$, and $C_{44} / \mathrm{GPa}$, and melting point $M_{p} / \mathrm{K}$.

\begin{tabular}{lccc}
\hline Label & Ref. [17-19] & Calculation & Error \\
\hline$a$ & 4.05 & 4.032 & $4 \%$ \\
$C_{11}$ & 114.7 & 113.7 & $0.87 \%$ \\
$C_{12}$ & 61.24 & 61.7 & $0.75 \%$ \\
$C_{44}$ & 32.86 & 31.2 & $2 \%$ \\
$M_{p}$ & 933.47 & 1023.7 & $9.6 \%$ \\
\hline
\end{tabular}

2.4. Definitions of Atomic Stress and Strain. In the molecular dynamics simulation, the calculation of stress at atomic scale is different from that at macroscopic scale. The atomic stress should meet the requirements of Viral condition, which can be described as the following form:

$$
\begin{aligned}
\sigma_{\alpha \beta} & =\frac{1}{\Omega} \sum_{i=1}^{N}\left(-m^{(i)}\left(u_{\alpha}^{(i)}-\bar{u}_{\alpha}\right)\left(u_{\beta}^{(i)}-\bar{u}_{\beta}\right)\right. \\
& \left.+\frac{1}{2} \sum_{j \neq i}\left(x_{\alpha}^{(j)}-x_{\alpha}^{(i)}\right) f_{\beta}^{i j}\right),
\end{aligned}
$$

where $\alpha$ and $\beta$ are the directions of the stress tensor, $i$ and $j$ are atoms in the system, $N$ is the atom number, $\Omega$ is the volume of system, $m$ is the atomic mass of the $i$ th atom, and $u_{\alpha}$ and $u_{\beta}$ represent the velocities along different directions. $\sigma_{\alpha \beta}$ is the average stress of the system, which mainly consists of two parts, namely, the velocity item and the displacement item. The velocity item represents the contribution of kinetic energy to the atom, and the displacement item represents the contribution of force to the atom, where the force is the one applied on the $i$ th atom by the other atoms. The strain is the ductility of single-crystal nickel in Potirniche simulation under tensile load [14]:

$$
\varepsilon_{y}=\frac{\left|L_{y_{0}}-L_{y}\right|}{L_{y_{0}}},
$$

where $L_{y_{0}}$ and $L_{y}$ are the initial model length and total length in $y$ direction, respectively.

\section{Results and Discussions}

3.1. Effects of Stress-Strain Curve. The stress-strain relationships during the tensile processes of single-crystal aluminum with four model sizes (atom number being 6704, 26136,
103304, and 410760, resp.) are shown in Figure 3. In the stage of plastic deformation, with the increase in the model size, the limit yield stress and the corresponding yield strain of the material decrease and the plastic yield point moves forward. When the atom number is 6704 , the limit stress of material reaches $6.98 \mathrm{GPa}$, and when the atom number is 410760 , the yield stress is $5.1 \mathrm{GPa}$; thus the plastic deformability of the material increases. It is attributed to the fact that, with the increase in the atom number, the percentage of surface atoms to all atoms decreases, the constraint on internal atoms of the system by surface stress decreases (i.e., the surface effect weakens), and the resistance toward dislocation propagation inside the system also decreases. This results in the phenomena of plastic yield strength.

Compared to the models with relatively large atom scale ( $\mathrm{N}$ is 103304 and 410760 , resp.), relatively obvious fluctuation occurs at the stress-strain curves after plastic flow in the small-scale models ( $N$ is 6704 and 26136, resp.). For smallerscale models, the space and freedom for local atomic motion are relatively small, resulting in small local atomic interactions. With the increase in the atoms scale, larger space is provided for motion, propagation, and interaction of the dislocations. Moreover, the atomic stress follows the statistical law; that is, the stress oscillation is averaged by a mass of atoms to decrease at microscales in the case of large atom number. With the increase in the model size, the decreasing speeds of the plastic yield stress and corresponding yield strain decrease. The size effect decreases gradually with the continued increase in size.

3.2. Effect of Model Size on Crack Propagation. Under high temperature and high strain rate conditions, formation of microscopic defects such as vacancies, dislocations, twin crystals, and slippages [26] in single-crystal aluminum material becomes easy. The formation, multiplication, and interaction of these microscopic defects affect the mechanical properties of metal materials such as plastic deformation, damage, and fracture.

The typical atomic configuration (Figures $4(\mathrm{~b})-4(\mathrm{e})$ ) of the stress-strain curve (Figure 4(a)) during the tensile process of SCAP with model 1 (number $N=6704$ ) is shown in Figure 4. After adequate relaxation of the model, the material is of FCC structure; however, because of the existence of the preset crack, dislocation nucleation is observed in the atomic system, as shown in Figure 4(b). When the material reaches the yield limit under load, the crack tip emits dislocations along the $\langle 110\rangle$ orientation and the crack becomes passivated and propagates with ductility, as shown in Figure 4(c). When the load is continued to increase, the dislocation nucleates at 


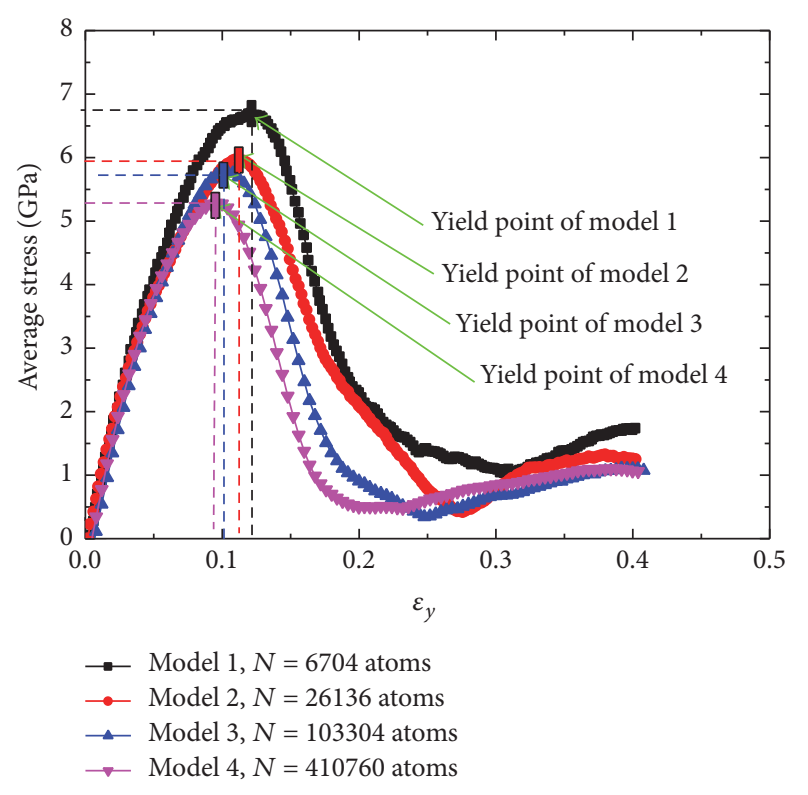

FIGURE 3: The stress-strain relationship for different atomic numbers, with the number $(N)$ of atoms being 6704 (model 1), 26136 (model 2), 103304 (model 3), and 410760 (model 4), respectively.

several sites on free surface and slips along the $\{111\}\langle 110\rangle$ slip system; the accumulated energy resulting from tension of SCAP is released due to the motion of the dislocation, and the stress decreases rapidly, as shown in Figure 4(d). Owing to the limited space for dislocation slipping at small scale, the entire slip plane starts to move, and the crack continues to propagate with ductility, as shown in Figure 4(e).

In the process of stretching, no obvious microcracks or microvoids are formed due to the larger specific surface area per volume at small scale, which is good for atom slipping; therefore, no microcrack is generated.

Figure 5 shows the stress-strain curve during tensile process (Figure 5(a)) and the corresponding typical atomic configuration (Figures 5(b)-5(e)) of model 4. When the load is applied to model, the crack tip emits dislocations along $\langle 110\rangle$ orientation group and microcrack is formed, as shown in Figure 5(b). When the loading stress reaches the limit strength, a mass of dislocations piles up around the crack and the crack slips along the $\langle 111\rangle$ slip plane. Further, the stress decreases rapidly due to the massive slippage of atoms, and the crack propagates with ductility, as shown in Figure 5(c). A large area of dislocations is formed with the continued multiplication and motion of dislocations, and the dislocation area at boundary exhibits an antipileup effect on the crack tip. This leads to the formation of a dislocation-free region near the crack tip, where stress concentrates and crack propagates rapidly, as shown in Figure 5(d). After the stress reaches the minimum point, it starts increasing with the further increase in the strain, resulting in the plastic hardening of material. Moreover, a large area of twin crystal appears along [110] orientation, which enhances the capability of material to resist deformation, and the stress increases with strain, as shown in Figure 5(e).
Comparison of Figures 4 and 5 indicates that the mechanisms for plastic deformation and crack propagation in SCAP at different scales are different. At small scale $(N=6704)$, the dislocation nucleation occurs first due to the preset crack and load, and this result is similar to the results of singlecrystal copper under tensile load as reported by Tschopp et al. [27]. With the increase in load, the lattice distortion occurs for FCC structure, and when the stress reaches the critical stress for dislocation emission, the dislocation emission and multiplication start. The emission and multiplication of the dislocation result in stress concentration, and when the stress reaches the critical stress for atom slipping, atom slips along the slip system, leading to rapid decrease of stress. At small scale, the dislocation nucleation and atom slipping play major roles. At large scale $(N=410760)$, the results after the relaxation of model are similar to those at small scale; however, occurrence of more dislocation nucleation is observed at the initial configuration. The larger scale of models provides larger space and more opportunity for dislocation nucleation. When the stress reaches the value for dislocation emission under load, the dislocation emission and multiplication occur. Nonetheless, due to the large scale, the dislocation cannot be emitted to the boundary and it piles up inside the material to form dislocation region. The plastic deformation is dominated by dislocation slip and twinning deformation. Noteworthily, dislocation-free region is formed at large scale and this result is similar to the fracture failure results of silicon alloy under impulse load [28].

During the process of crack propagation, a new structure is formed due to the motion and pileup of dislocations. Figure 6 exhibits the atomic structure and stress nephogram of model 4 when the atomic configuration changes from FCC structure to hexagonal close-packed (HCP) structure. Before the transition to HCP structure, the stress near the crack tip is highly concentrated, and after the formation of the HCP structure (Figure 6(a)), the stress in this area decreases consequently. Through phase transition, the stress in the crystal structure can be released, which leads to the decrease in the stress in the phase transition region, as shown in Figure 6(b).

Noteworthily, the stress concentration at the crack tip is the fundamental reason for the phase transition at the crack tip from FCC to HCP. The stress concentration induces severe distortion of the FCC structure, leading to rearrangement of the atoms to form the HCP structure, which is in well agreement with the conclusion that the phase transition is induced by stress concentration as demonstrated by Nishimura and Miyazaki [29] and Nishimura and Miyazaki [30] through simulation and experimental methods.

The stress at yielding of the material is yield stress; its plastic yield is related to the slippage number and type. Figure 7 shows the atomic configurations corresponding to the plastic yields for atom numbers 6704, 26136, 103304, and 410760 (Figures 7(a), 7(b), 7(c), and 7(d), resp.). When the material is yielding, the slip systems of model $1(N=6704)$ are $[\overline{1} 10](\overline{1} 11)$ and $[110](111)$, as shown in Figure $7(\mathrm{a})$. The slip systems of model $2(N=26136)$ are [ $\overline{1} 10](111)$, [1 $\overline{1} 0](111)$, and $[\overline{1} \overline{1} 0](\overline{1} \overline{1} 1)$, as shown in Figure $7(\mathrm{~b})$. The slip systems of model $3(N=103304)$ are [110](111), [ $\overline{1} 10](111)$, $[\overline{1} \overline{1} 0](1 \overline{1} 1), \quad[1 \overline{1} 0](\overline{1} \overline{1} \overline{1})$, and $[1 \overline{1} 0](11 \overline{1})$, as shown in 


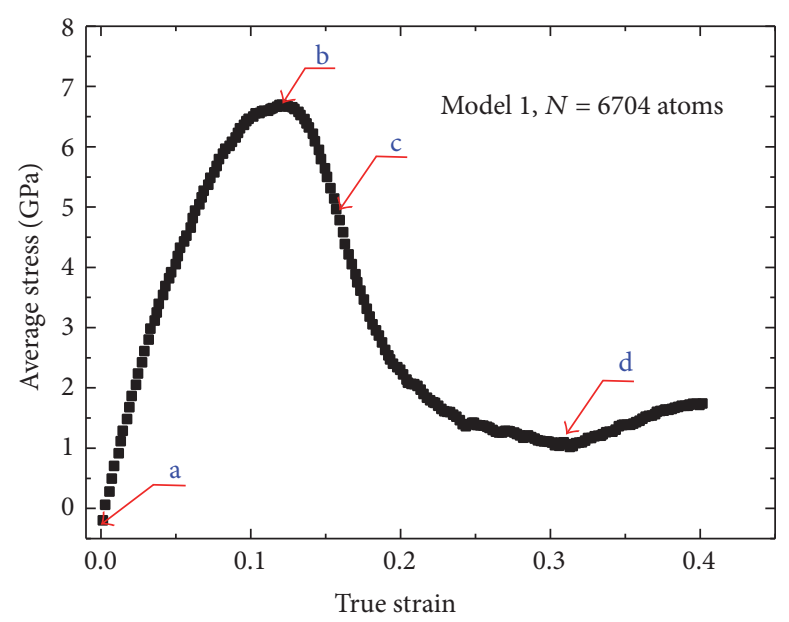

(a)

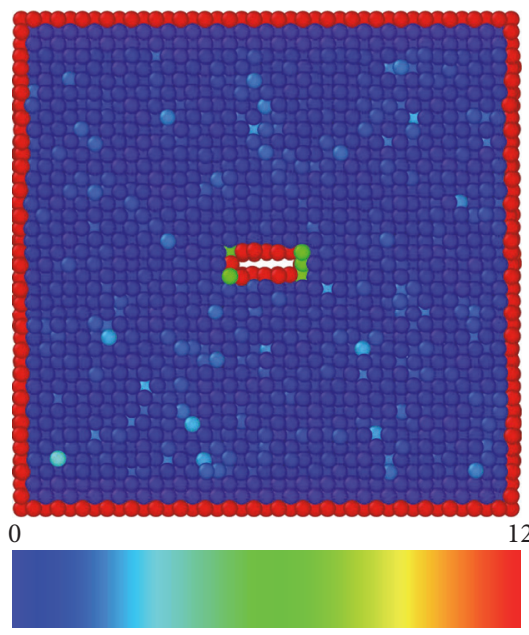

(b)

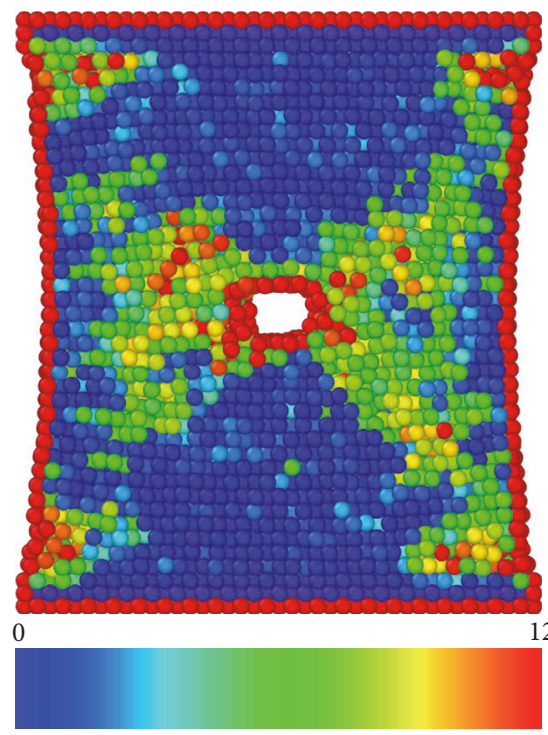

(d)

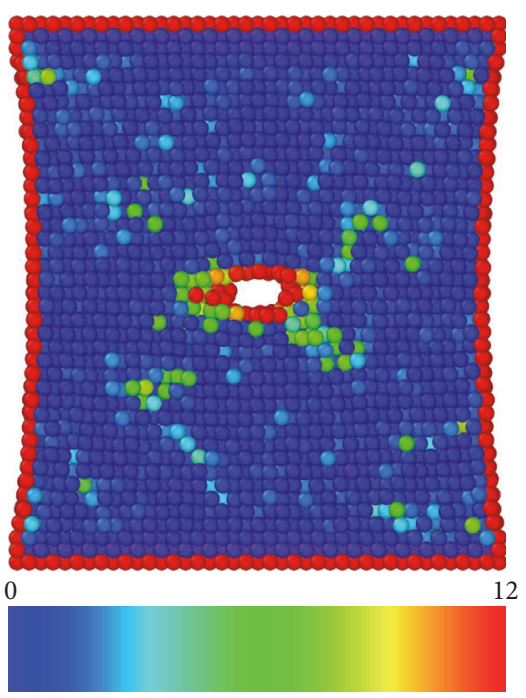

(c)

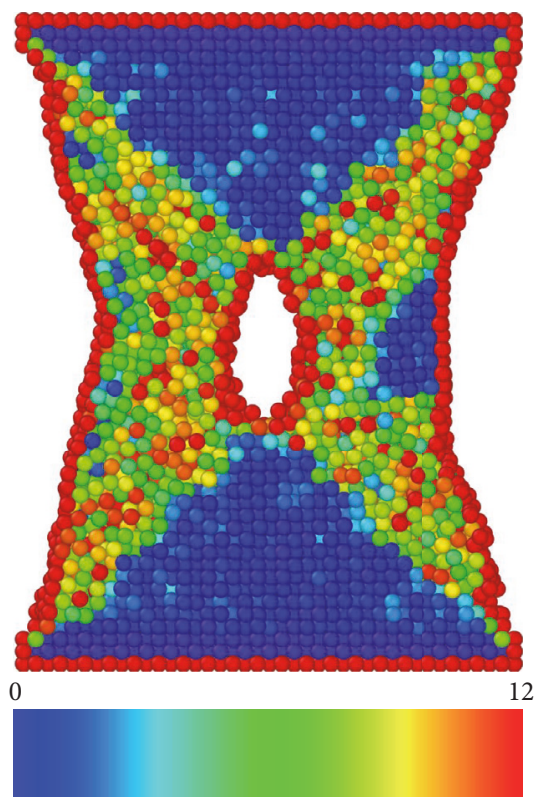

(e)

Figure 4: (a) The stress-strain relationship for the model of 6704 atoms (model 1); (b)-(e) are the atomic configuration corresponding points a-d in (a), respectively. 


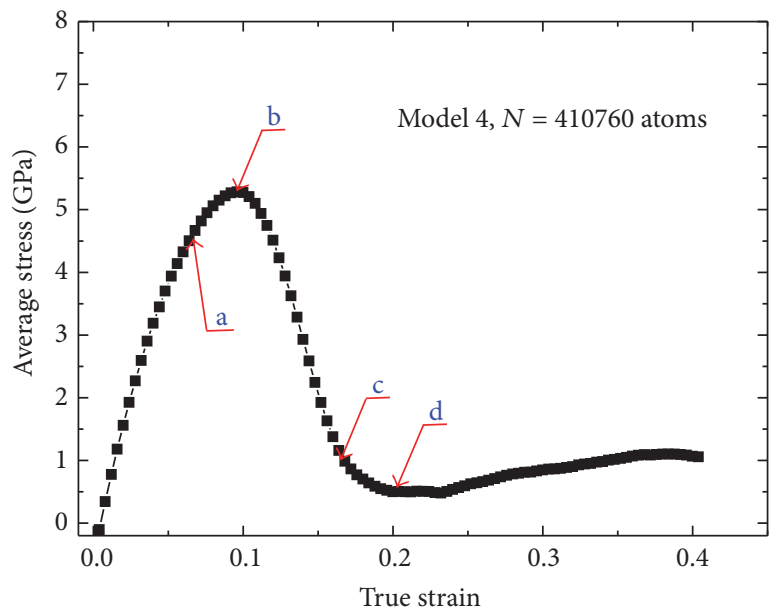

(a)

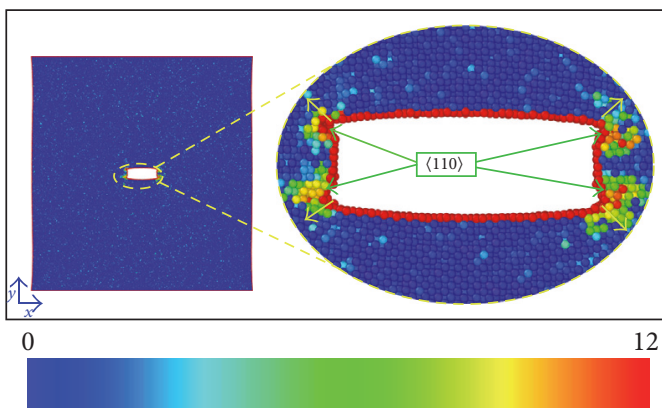

(b)

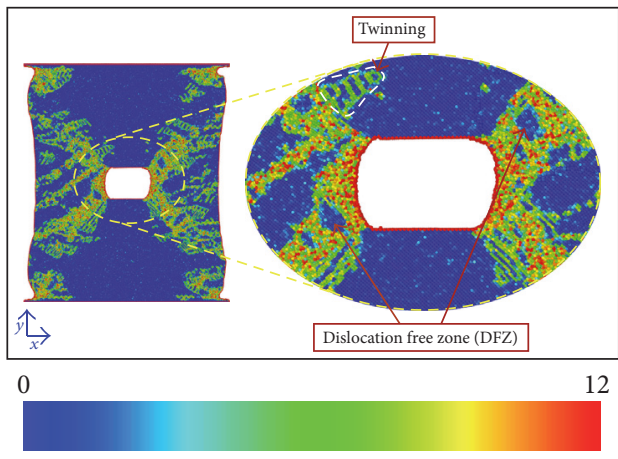

(d)

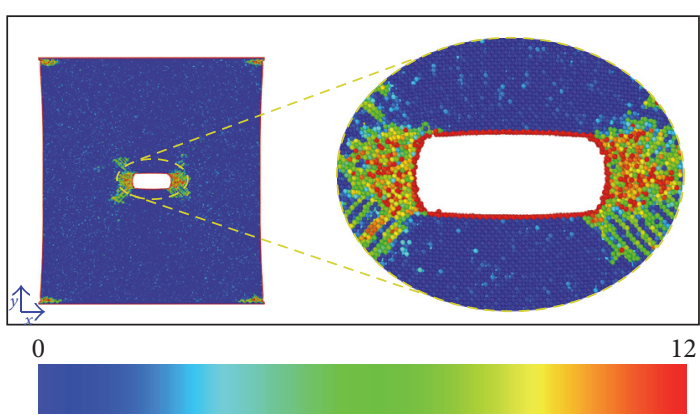

(c)

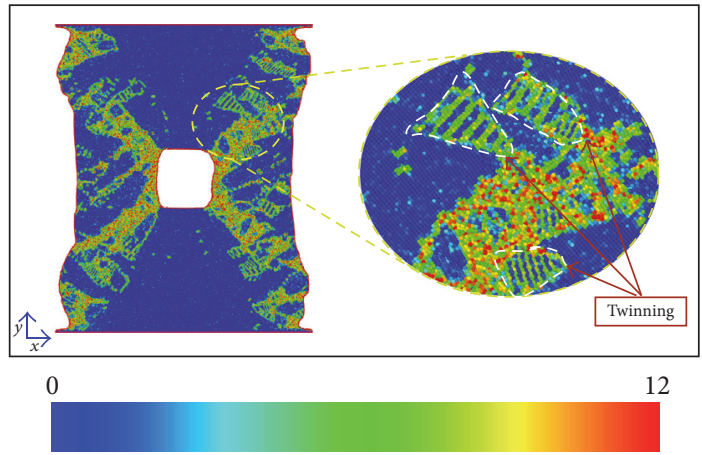

(e)

FiguRE 5: (a) The stress-strain relationship for the model of 410760 atoms (model 4); (b)-(e) are the atomic configuration corresponding points a-d in (a), respectively.

Figure $7(\mathrm{c})$. The slip system of model $4(N=410760)$ is $\{111\}\langle 110\rangle$, as shown in Figure $7(\mathrm{~d})$. The different model size results in different number of staring slip systems at the yield stress. The number of the triggered slip system increases, and the plastic deformability of material is enhanced; lower stress is needed for yielding of material, and the yield strength is decreased. Model 3 generates double slip along

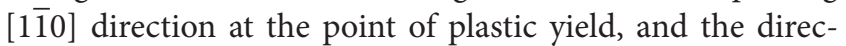
tions of the double slip do not interact with each other. In contrast, the double slip facilitates the slip, thus reducing the yield stress.
The strength of material at yield stress is also related to the dislocation density. The increase in model size leads to an increase in the dislocation density and decrease in the yield strength. With the increase in the model size, the restriction of geometrically necessary dislocations on the statistical dislocations decreases, and the space and probability of the dislocation nucleation increase, which results in the decreased probability for local nonuniform plastic deformation. This result is consistent with the results of yield strength of material decreasing with model size as reported by Geers et al. [31]. 

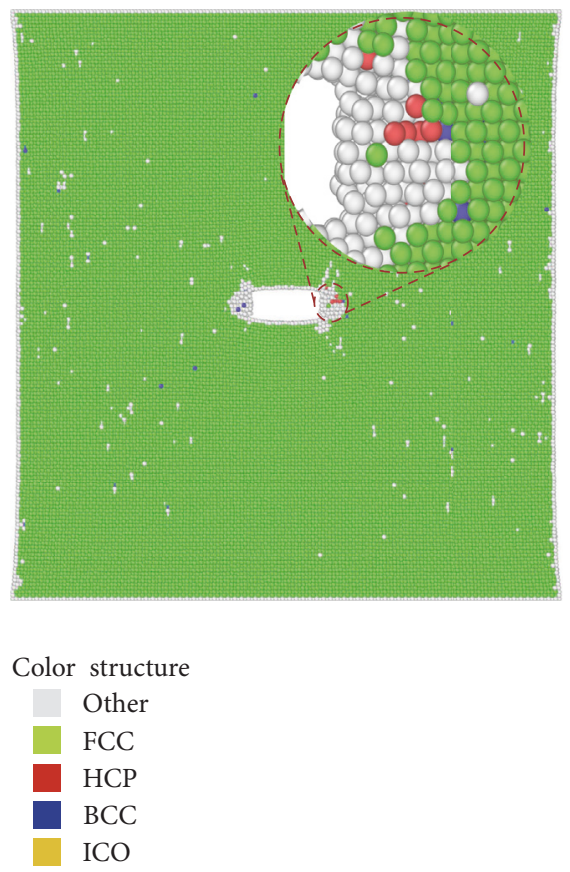

(a)

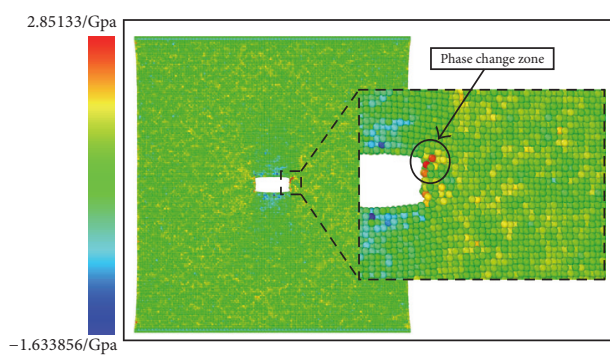

(b)

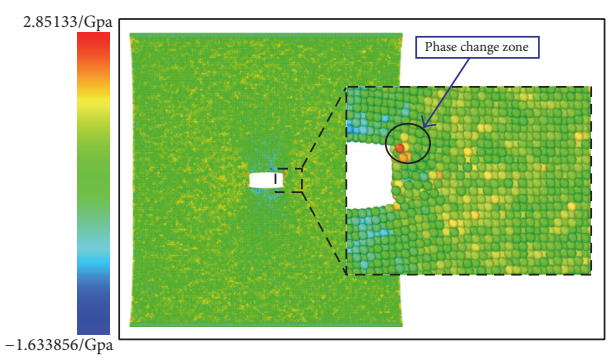

(c)

Figure 6: (a) The atomic structure after phase transition for 103304 atoms (model 3). (b) The stress contour near the cracks for the model 3 before transition. (c) The stress contour near the cracks for the model 3 before transition, respectively.

3.3. Effect of Crack Length on Stress-Strain Relationship. The stress-strain relationship of SCAP during the tensile process is shown in Figure 8. Clearly, the changes in the stressstrain curves of the models with different crack lengths are basically consistent. When the material reaches the plastic flow conditions, the plastic yield stress decreases and the yield point is attained in advance with the increasing crack length. When reaching the yield limit, the stress is sufficient for triggering the slip systems. The atoms inside the system slip massively, and the stress decreases rapidly to the minimum. However, the decreasing range of relatively small crack length is the largest and increasing crack length aids in increasing plastic deformability.

The yield stress for the three crack lengths is 5.7, 5.1, and $4.5 \mathrm{GPa}$, respectively. With the increase in the crack length, the yield stress decreases, the external load required for plastic flow of material decreases, and the plastic deformation of the material is enhanced. According to the energy conservation, the shorter the preset crack length, the lower the atomic potential between the cracks and the higher the remaining atomic potential, and the stress required for plastic flow of crack is increased further.

The yield strength influences the number of staring slip systems. Figure 9 exhibits the atomic configurations at the plastic yield point for crack lengths of 2.592, 3.888, and $6.480 \mathrm{~nm}$, respectively. When the plastic yield limit is attained, the starting slip systems of atomic system for crack length of $2.592 \mathrm{~nm}$ are $[1 \overline{1} 0](\overline{1} 11),[\overline{1} 10](\overline{1} 11)$, and

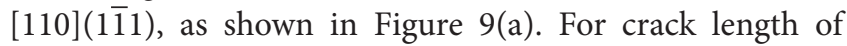
$3.888 \mathrm{~nm}$, the dislocations slip along slip systems corresponding to $[110](\overline{1} 11),[\overline{1} 10](111),[\overline{1} \overline{1} 0](1 \overline{1} 1),[1 \overline{1} 0](\overline{1} \overline{1} \overline{1})$, and $[1 \overline{1} 0](11 \overline{1})$, as shown in Figure 9(b). For crack length of $6.480 \mathrm{~nm}$, the dislocations slip along slip systems of $[110](\overline{1} 11), \quad[\overline{1} \overline{1} 0](\overline{1} \overline{1} 1), \quad[1 \overline{1} 0](\overline{1} \overline{1} \overline{1}), \quad[\overline{1} 10](111)$, and $[\overline{1} 10](11 \overline{1})$, as shown in Figure 9(c).

The crack length affects the number of starting slip systems. When the plastic yield of the material occurs, the number of the starting slip systems increases with the increase in the crack length, and the limit yield stress decreases. When the crack length is relatively small, the dislocation pileup 


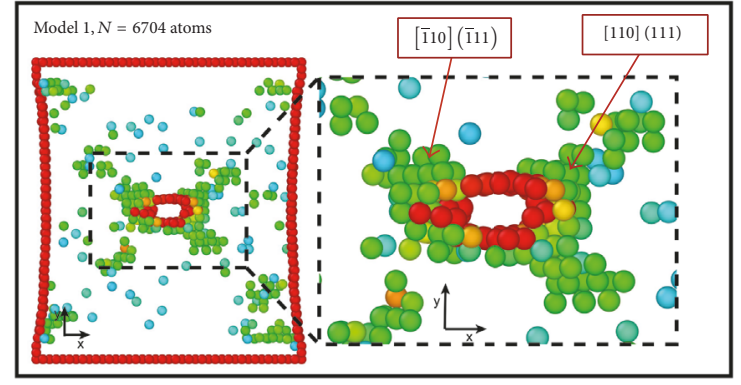

(a)

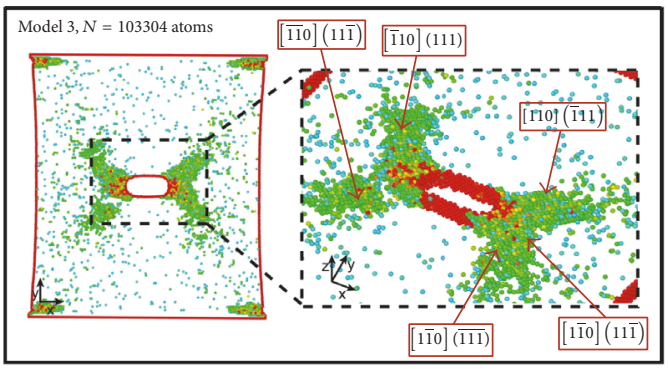

(c)

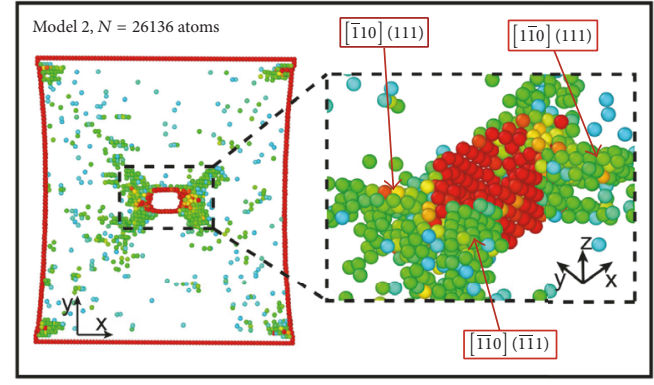

(b)

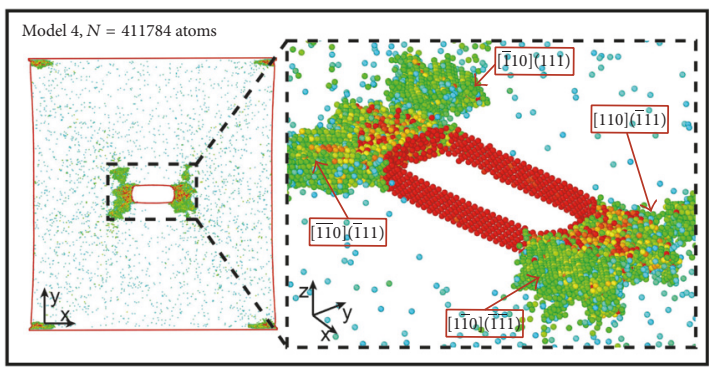

(d)

Figure 7: The slip systems for different atomic numbers in plastic yield point. (a) $N=6704$ (model 1); (b) $N=26136$ (model 2); (c) $N=103304$ (model 3); and (d) $N=410760$ (model 4).

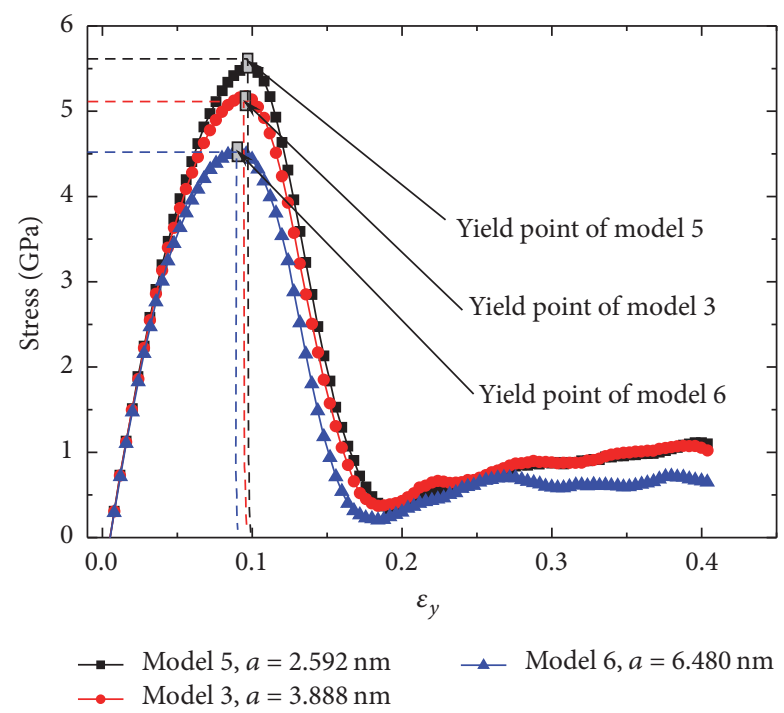

FIGURE 8: The stress-strain relationship for different crack lengths of $2.592 \mathrm{~nm}$ (model 5), $3.888 \mathrm{~nm}$ (model 3), and $6.480 \mathrm{~nm}$ (model 6), respectively.

region is basically disordered. For crack length of $3.88 \mathrm{~nm}$, the dislocations pile up mainly along the loading directions [010] and $[0 \overline{1} 0]$. For crack length of $6.480 \mathrm{~nm}$, the dislocations pile up mainly along the $\langle 010\rangle$ orientation.

With the increase in the crack length, the dislocations pileup plugged along the direction of the slip of the single crystal; thus it was easier for the crystal to slip and move, and the plasticity of the material was enhanced. The strength of the material during plastic yielding was also related to the dislocation density. With the increase in the crack length, the dislocation density increased and the yield strength decreased.

3.4. Effect of Temperature Strain Rate on Stress-Strain Relationship. The stress-strain relationships of SCAP during the tensile process at various temperatures are shown in Figure 10. When the plastic hardening of the material occurs, the corresponding yield limit decreases with relatively small effects, and the plastic deformation point moves forward. With the increase in the temperature, the internal kinetic energy increases, and the atomic motion is enhanced. The temperature sensitivity of the material is relatively low, the dislocation width of FCC structure is relatively large, and the influences of temperature on the capability of the material to resist deformation and plastic yield strength are relatively small, and these results are consistent with the results reported by Brochard et al. [32].

After the material reaches its yield point, the stress decreases rapidly to the minimum with the strain, and at relatively high temperature $(900 \mathrm{~K})$, the corresponding minimum stress is $0.13 \mathrm{GPa}$. After the stress decreases to the lowest point, it starts increasing with the strain, resulting in plastic hardening of the material. After the stress decreases to its minimum value, with the increasing temperature, the stress-strain curve turns to be more rugged. At the nanoscale, existence of the heat stress is observed between the atoms, and the higher the temperature, the more obvious the heat stress.

Figure 11 shows the stress-strain relationships for various loading strain rates $\left(10^{8}, 10^{9}\right.$, and $10^{10} \mathrm{~s}^{-1}$, resp.). When the strain rate is increased by orders, the yield limit stress 


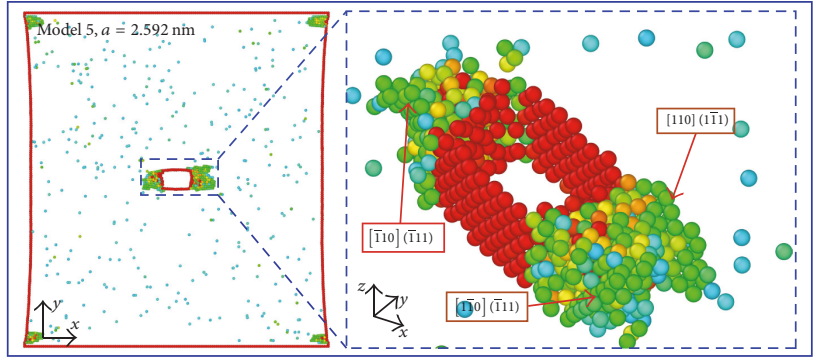

(a)

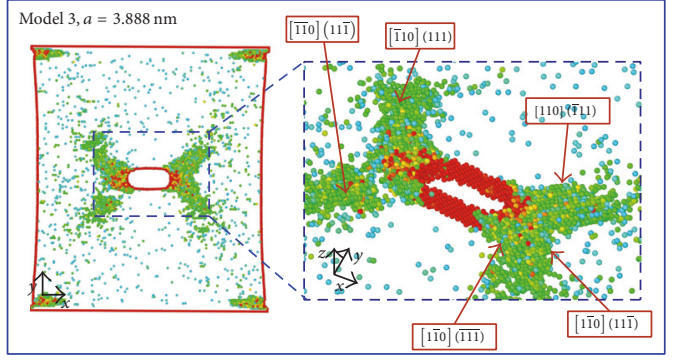

(b)

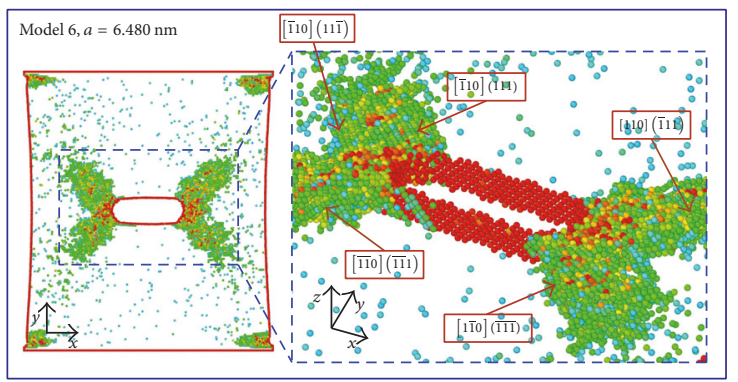

(c)

Figure 9: The slip systems for different crack lengths: (a) $a=2.592 \mathrm{~nm}$ (model 5), (b) $a=3.888 \mathrm{~nm}$ (model 3), and (c) $a=6.480 \mathrm{~nm}$ (model $6)$.

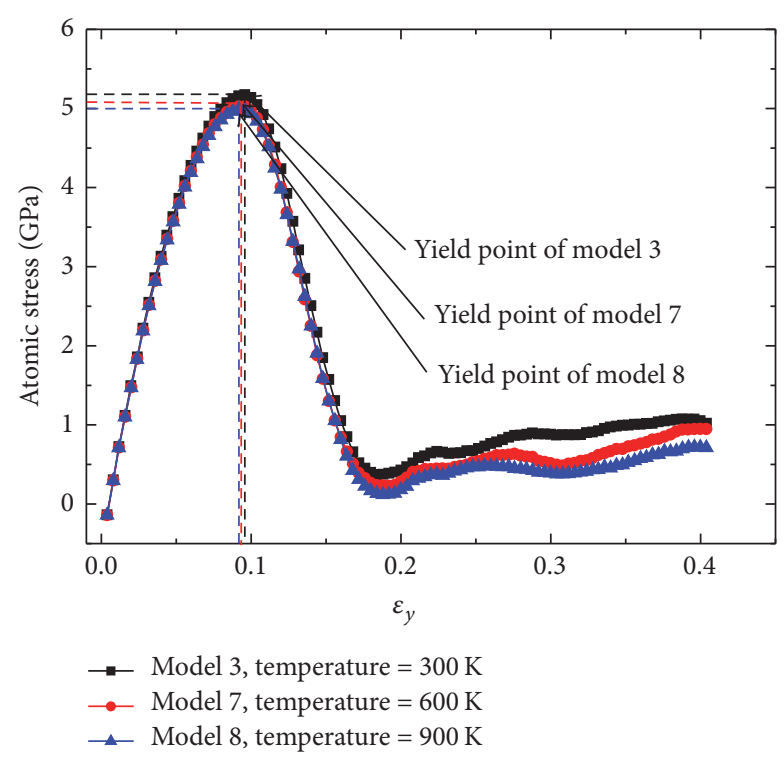

FIGURE 10: The stress-strain relationship for different temperatures of $300 \mathrm{~K}$ (model 3), $600 \mathrm{~K}$ (model 7), and $900 \mathrm{~K}$ (model 8), respectively.

increases from 3.72 to $6.35 \mathrm{GPa}$, and the plastic deformability of material is enhanced. Under relatively low strain rate, the starting of slip bands in atomic system occurs at nearly the same time. However, with the increasing loading velocity, the dislocation motion is hindered by the dynamic fluctuations and phonon lag, and the slip band needs higher stress in a small area to propagate inside the model.
When the stress reaches the yield limit, the peak stress is relatively obvious for relatively low strain rate $\left(10^{8} \mathrm{~s}^{-1}\right)$, and the peak stress is not obvious for relatively high strain rate $\left(10^{10} \mathrm{~s}^{-1}\right)$, with no obvious yield point. The increase in strain rate induces a transition region between the stage of elastic deformation and the stage of plastic deformation. After reaching the yield limit, the stress decreases rapidly with the strain; however, the decreasing rate for relatively low loading velocity is smaller than that for relatively high loading velocity.

After the stress decreases to its lowest point, the oscillation amplitude of stress-strain curve decreases with the strain rate, and the plastic hardening becomes more obvious. For low strain rate, the kinetic energy of the atoms in system is relatively small, and the capability toward atom slip is relatively low. However, under high strain rate, the kinetic energy increases significantly, and the capability for slip increases, thus promoting the plastic deformability of the material.

\section{Conclusions}

In this study, the fracture failure process of nanoaluminum plate with central crack under the uniaxial tension was simulated by using molecular dynamics method, and the main conclusions were drawn as follows.

(1) At the nanoscale, the model of SCAP with central crack exhibited typical size effect. With the increase in the model size, the plastic yield point was attained in advance, the limit stress of the plastic yield decreased, and the plastic deformability of material increased. The stress concentration at crack tip was the fundamental reason for the structure 


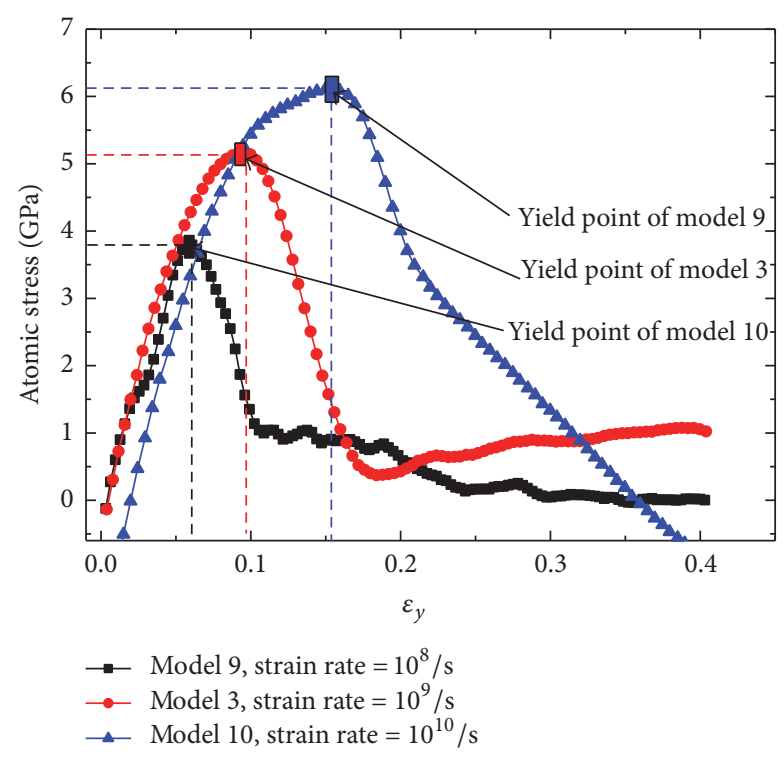

FIgURE 11: The stress-strain relationship for different strain rates of $10^{8} \mathrm{~s}^{-1}$ (model 9), $10^{9} \mathrm{~s}^{-1}$ (model 3), and $10^{10} \mathrm{~s}^{-1}$ (model 10), respectively.

transition at crack tip, and the stress of structure was found to be relaxed after the transition. The stress decreased with strain, and when the stress reached the minimum, the plastic hardening occurred, and the dependence of strength of SCAP on size was weakened.

(2) The crack propagation mechanism at different scales was different. At relatively small scale, the plastic deformation and crack growth occurred in SCAP following the mechanism of "dislocation nucleation-dislocation emission and multiplication-cross-slip of dislocation and lattice atom." However, at relatively large scale, the crack growth and fracture failure are dominated by dislocation slip and twinning deformation.

(3) With different model size, the number of staring slip systems when reaching yield limit was different. With the increasing number of starting slip systems, the plastic deformability of material increased, the stress required for material yield decreased, and the yield strength also decreased. The strength at the plastic yield point was also related to the dislocation density; with the model size increasing, the dislocation density increased and the yield strength decreased.

(4) At the nanoscale, the crack length significantly affected the strength of SCAP. After the yield limit, the plastic yield point decreased with crack length, and the plastic yield point was attained in advance. Further, the plastic deformability of material increased. The crack length influenced the stress for plastic flow through the atomic potential between cracks. At the plastic yield point, with the increasing crack length, the number of staring slip systems increased, and the limit yield stress decreased. The dislocation pileup direction was the same as the single-crystal slip direction, which was beneficial to the slip and motion of crystal, resulting in the enhanced plastic ability of material.
(5) The effect of temperature on the strength of SCAP was relatively small, and the temperature sensitivity of the material was also relatively low. At the nanoscale, the heat stress existed between the atoms, and the higher the temperature, the more obvious the heat stress. Under high strain rate loading, with the increase in the loading strain rate, the yield limit increased, and the plastic deformation point was attained in advance; the plastic deformability of material increased. After the yield limit, the stress decreased rapidly with strain, and the decreasing rate for relatively low loading velocity was smaller than that for relatively high loading velocity.

\section{Conflicts of Interest}

The authors declare that there are no conflicts of interest regarding the publication of this paper.

\section{Acknowledgments}

This work is financially supported by NSAF (Grant no. U1530140) and by Chongqing Science and Technology Committee (Grant nos. CSTC2016JCYJA0517 and CSTC2017JCYJA0857).

\section{References}

[1] M. F. Horstemeyer, M. I. Baskes, and S. J. Plimpton, "Computational nanoscale plasticity simulations using embedded atom potentials," Theoretical and Applied Fracture Mechanics, vol. 37, no. 1-3, pp. 49-98, 2001.

[2] P. White, "Molecular dynamic modelling of fatigue crack growth in aluminium using LEFM boundary conditions," International Journal of Fatigue, vol. 44, pp. 141-150, 2012.

[3] Y. Q. Yuan, H. Y. Chen, X. G. Zeng, and Y. F. Hu, "MD simulation on evolution of micro structure and failure mechanism around interactional voids in pure al," Applied Mechanics and Materials, vol. 444-445, pp. 183-190, 2014.

[4] C. B. Cui and H. G. Beom, "Molecular dynamics simulations of edge cracks in copper and aluminum single crystals," Materials Science and Engineering A, vol. 609, pp. 102-109, 2014.

[5] H. P. Chen, "Deformation and micro fracture of granular bainite," ACTA Metallurgica Sinica, vol. 28, pp. 226-229, 1992.

[6] Y. Takahashi, T. Shikama, S. Yoshihara, T. Aiura, and H. Noguchi, "Study on dominant mechanism of high-cycle fatigue life in 6061-T6 aluminum alloy through microanalyses of microstructurally small cracks," Acta Materialia, vol. 60, no. 6-7, pp. 2554-2567, 2012.

[7] A. A. Shanyavskiy, "Mechanisms and modeling of subsurface fatigue cracking in metals," Engineering Fracture Mechanics, vol. 110, pp. 350-363, 2013.

[8] J. K. Mahato, P. S. De, A. Sarkar, A. Kundu, and P. C. Chakraborti, "Effect of deformation mode and grain size on Bauschinger behavior of annealed copper," International Journal of Fatigue, vol. 83, pp. 42-52, 2015.

[9] G. P. Potirniche, M. F. Horstemeyer, G. J. Wagner, and P. M. Gullett, "A molecular dynamics study of void growth and coalescence in single crystal nickel," International Journal of Plasticity, vol. 22, no. 2, pp. 257-278, 2006.

[10] D. J. Dunstan and A. J. Bushby, "The scaling exponent in the size effect of small scale plastic deformation," International Journal of Plasticity, vol. 40, pp. 152-162, 2013. 
[11] M. Yaghoobi and G. Z. Voyiadjis, "Size effects in fcc crystals during the high rate compression test," Acta Materialia, vol. 121, pp. 190-201, 2016.

[12] Y. Liao, M. Xiang, X. Zeng, and J. Chen, "Molecular dynamics studies of the roles of microstructure and thermal effects in spallation of aluminum," Mechanics of Materials, vol. 84, pp. 1227, 2015.

[13] B. Shiari and R. E. Miller, "Multiscale modeling of crack initiation and propagation at the nanoscale," Journal of the Mechanics and Physics of Solids, vol. 88, pp. 35-49, 2016.

[14] M. F. Horstemeyer, M. I. Baskes, and S. J. Plimpton, "Length scale and time scale effects on the plastic flow of fcc metals," Acta Materialia, vol. 49, no. 20, pp. 4363-4374, 2001.

[15] S. L. Yan, H. Yang, H. W. Li, and X. Yao, "Variation of strain rate sensitivity of an aluminum alloy in a wide strain rate range: Mechanism analysis and modeling," Journal of Alloys and Compounds, vol. 688, pp. 776-786, 2016.

[16] X.-B. Liu, Q.-J. Xu, and J. Liu, "Molecular dynamics simulation of crack propagation behavior of aluminum," The Chinese Journal of Nonferrous Metals, vol. 24, no. 6, pp. 1408-1413, 2014.

[17] K. Syassen and W. B. Holzapfel, "HIGH-PRESSURE-LOWTEMPERATURE X-RAY POWER DIFFRACTOMETER.," Review of Scientific Instruments, vol. 49, no. 8, pp. 1107-1111, 1978.

[18] S. Falconi, W. A. Crichton, M. Mezouar, G. Monaco, and M. Nardone, "Structure of fluid phosphorus at high temperature and pressure: an X-ray diffraction study," Physical Review B, vol. 70, no. 14, pp. 94-112, 2004.

[19] Y. Hiki and A. V. Granato, "Anharmonicity in noble metals; higher order elastic constants," Physical Review, vol. 144, no. 2, pp. 411-419, 1966.

[20] W. C. Swope, H. C. Andersen, P. H. Berens, and K. R. Wilson, "A computer simulation method for the calculation of equilibrium constants for the formation of physical clusters of molecules: application to small water clusters," The Journal of Chemical Physics, vol. 76, no. 1, pp. 637-649, 1982.

[21] C. L. Kelchner, S. J. Plimpton, and J. C. Hamilton, "Dislocation nucleation and defect structure during surface indentation," Physical Review B, vol. 58, no. 17, pp. 11085-11088, 1998.

[22] D. Faken and H. Jónsson, "Systematic analysis of local atomic structure combined with 3D computer graphics," Computational Materials Science, vol. 2, no. 2, pp. 279-286, 1994.

[23] H. Tsuzuki, P. S. Branicio, and J. P. Rino, "Structural characterization of deformed crystals by analysis of common atomic neighborhood," Computer Physics Communications, vol. 177, no. 6, pp. 518-523, 2007.

[24] J. M. Winey, A. Kubota, and Y. M. Gupta, "Erratum: Thermodynamic approach to determine accurate potentials for molecular dynamics simulations: Thermoelastic response of aluminum (Modelling Simul. Mater. Sci. Eng. (2009) 17 (055004)," Modelling and Simulation in Materials Science and Engineering, vol. 18, no. 2, Article ID 029801, 2010.

[25] J. M. Winey, A. Kubota, and Y. M. Gupta, "Thermo dynamic approach to determine accurate potentials for molecular dynamics simulations: thermoelastic response of aluminum," Modelling and Simulation in Materials Science and Engineering, vol. 18, no. 2, Article ID 029801, 2010.

[26] D. C. William and G. R. David, "Fundamentals of Materials Science and Engineering: An Integrated Approach [B]," pp. 2647, John Wiley and Sons, Newyork, NY, USA, 2011.
[27] M. A. Tschopp, D. E. Spearot, and D. L. McDowell, "Atomistic simulations of homogeneous dislocation nucleation in single crystal copper," Modelling and Simulation in Materials Science and Engineering, vol. 15, no. 7, pp. 693-709, 2007.

[28] H. Saka and Y. Agata, "Direct evidence for dislocation-free zone in Fe-5\% Si fractured by Charpy impact test," Materials Science and Engineering A, vol. 350, no. 1-2, pp. 57-62, 2003.

[29] K. Nishimura and N. Miyazaki, "Molecular dynamics simulation of crack propagation in polycrystalline material," Computer Modeling in Engineering and Sciences, vol. 2, no. 2, pp. 143-154, 2001.

[30] K. Nishimura and N. Miyazaki, "Molecular dynamics simulation of crack growth under cyclic loading," Computational Materials Science, vol. 31, no. 3-4, pp. 269-278, 2004.

[31] M. G. D. Geers, W. A. M. Brekelmans, and P. J. M. Janssen, "Size effects in miniaturized polycrystalline FCC samples: Strengthening versus weakening," International Journal of Solids and Structures, vol. 43, no. 24, pp. 7304-7321, 2006.

[32] S. Brochard, P. Hirel, L. Pizzagalli, and J. Godet, "Elastic limit for surface step dislocation nucleation in face-centered cubic metals: Temperature and step height dependence," Acta Materialia, vol. 58, no. 12, pp. 4182-4190, 2010. 

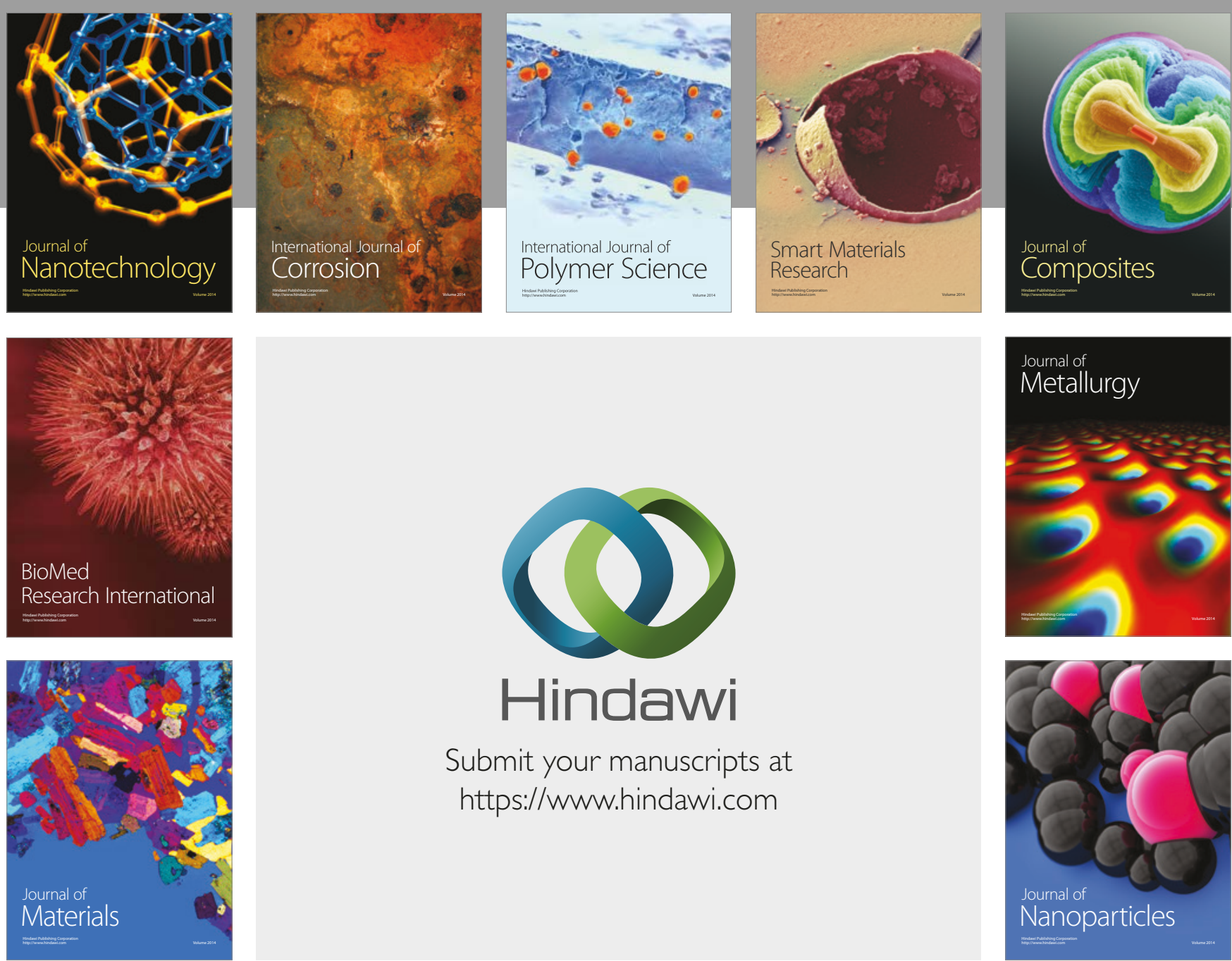

\section{Hindawi}

Submit your manuscripts at

https://www.hindawi.com
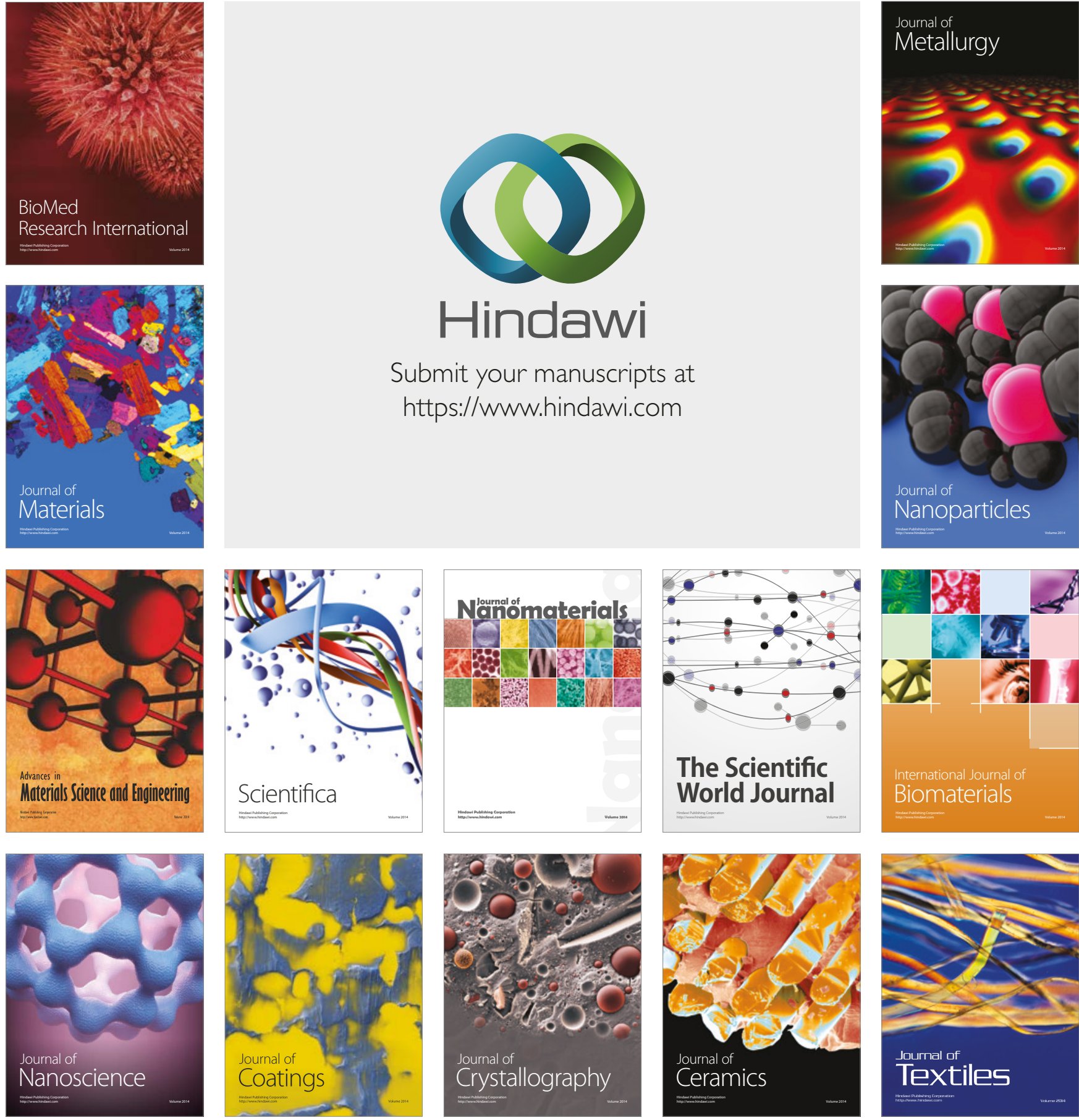

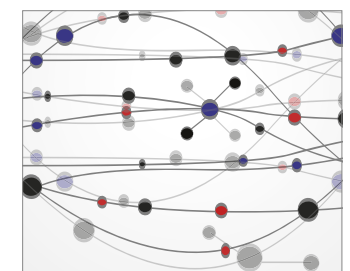

The Scientific World Journal
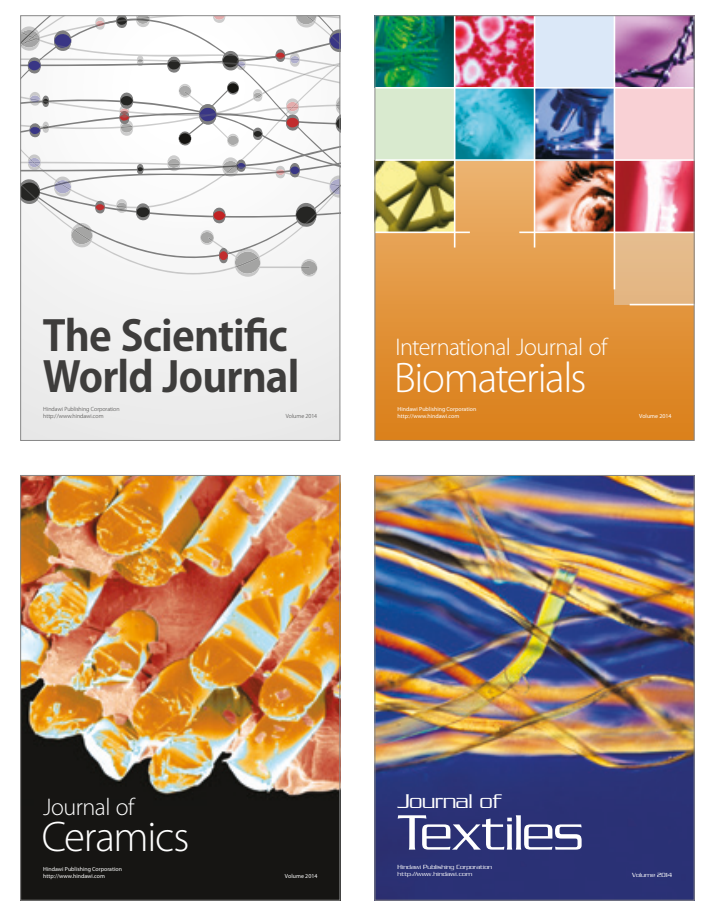